\title{
Perfil clínico de pacientes chagásicos e não-chagásicos portadores de marca-passo cardíaco artificial
}

\author{
Clinical profile of Chagas and non-Chagas' disease patients \\ with cardiac pacemaker
}

\author{
Leonor Garcia Rincon ${ }^{1,2}$, Manoel Otávio da Costa Rocha ${ }^{1}$, Marco Túlio Baccarini Pires ${ }^{2}$, \\ Bruna Guimarães Oliveira'2, Vladimir da Costa Val Barros ${ }^{3}$, \\ Marcio Vinicius Lins Barros ${ }^{3}$ e Antônio Luiz Pinho Ribeiro ${ }^{1,2}$
}

\begin{abstract}
RESUMO
O objetivo do estudo foi comparar pacientes chagásicos e não-chagásicos com marca-passo cardíaco artificial uni ou bicameral quanto à fração de ejeção do ventrículo esquerdo, o limiar de estimulação ventricular e a incidência de arritmias cardíacas. Entre janeiro/2001 e novembro/2002 foram avaliados 45 pacientes chagásicos e 35 não-chagásicos portadores de marcapasso quanto à história clínica, radiografia do tórax, ecocardiograma, Holter $24 \mathrm{~h}$ e análise telemétrica do marca-passo. Embora os chagásicos fossem mais jovens, os dois grupos foram semelhantes quanto a variáveis radiológicas e o limiar de estimulação ventricular direito. Os chagásicos apresentaram menor fração de ejeção do ventrículo esquerdo ao ecocardiograma e maior incidência de arritmia ventricular ao Holter $24 \mathrm{~h}$. Observou-se correlação positiva entre a baixa fração de ejeção do ventrículo esquerdo e a intensidade da arritmia ventricular ao Holter 24h. Em pacientes com marca-passo, a doença de Chagas está associada a marcadores cardíacos de prognóstico adverso.
\end{abstract}

Palavras-chaves: Doença de Chagas. Marca-passo. Arritmia. Telemetria.

\begin{abstract}
The aim of this study was to compare Chagas and non-Chagas' disease patients using single or dual-chamber pacemaker in relation to the ejection fraction of the left ventricle, the ventricular stimulation threshold and the occurrence of ventricular arrhythmia. From January, 2001 to November, 2002, 45 Chagas' disease patients and 35 non-Chagas' disease patients, all pacemaker users, were evaluated considering clinical history, echocardiographic study, Holter monitoring and analysis of the pacemaker telemetry data. Chagas' disease patients were significantly younger, but both groups were similar when chest X-Ray variables and right ventricular stimulation threshold were analyzed. Chagas' disease patients had a lower left ventricular ejection fraction and more frequent ventricular arrhythmia during Holter monitoring. A positive correlation between the low ejection fraction of the left ventricle and the intensity of ventricular arrhythmia was observed. In conclusion, among pacemaker user patients, Chagas' disease is related to cardiac markers of worse prognosis.
\end{abstract}

Key-words: Chagas' disease. Pacemaker. Arrhythmia. Telemetry.

A doença de Chagas é uma patologia endêmica, de larga distribuição nas Américas Central e do Sul. A doença é encontrada em 21 países deste continente, com cerca de16 a 18 milhões de pessoas infectadas e cem milhões em risco de adquiri-la $a^{21}$. A miocardite crônica fibrosante que ocorre na fase crônica da doença é responsável não só pela perda da função contrátil do miocárdio como também pelo comprometimento do sistema de condução do coração, por variados tipos de arritmias e sua associação não rara com a morte súbita ${ }^{13}$. A importância do bloqueio atrioventricular na doença de Chagas foi reconhecida desde os estudos iniciais de Chagas e Villela ${ }^{7}$, constituindose ainda hoje uma das mais freqüentes indicações para implante de marcapasso no Brasil.

\footnotetext{
1. Curso de Pós-Graduação em Ciências da Saúde, Infectologia e Medicina Tropical da Faculdade de Medicina da Universidade Federal de Minas Gerais, Belo Horizonte, MG, Brasil. 2. Serviço de Cardiologia do Hospital das Clínicas da Universidade Federal de Minas Gerais, Belo Horizonte, MG, Brasil. 3. Ecoar - Medicina Diagnóstica, Belo Horizonte, Brasil. Financiado em parte por auxílios do Conselho Nacional do Desenvolvimento Científico e Tecnológico (CNPq) e Fundação de Amparo à Pesquisa do Estado de Minas Gerais (FAPEMIG) Endereço para correspondência: Dr. Antonio L.P. Ribeiro. R. Campanha 98/101, 30310-770 Belo Horizonte, MG, Brasil.

Tel: 5531 3287-9213; Fax: 5531 3284-7298

e-mail: tom@hc.ufmg.br

Recebido para publicação em 19/7/2005

Aceito em 31/3/2006
} 
Estudos comparativos da cardiopatia chagásica com outras cardiopatias dilatadas revelam que pacientes chagásicos apresentam pior prognóstico ${ }^{16}{ }^{18}$. No entanto, a adequada caracterização do perfil funcional do paciente chagásico portador de marca-passo ainda não foi bem definida. Este estudo tem como objetivo comparar pacientes chagásicos e não-chagásicos com marca-passo cardíaco artificial quanto à fração de ejeção do ventrículo esquerdo obtida pela ecodopplercardiografia, ao limiar de estimulação ventricular através da telemetria e à incidência de arritmias cardíacas registradas ao Holter $24 \mathrm{~h}$.

\section{MATERIAL E MÉTODOS}

Foram selecionados oitenta pacientes portadores de marca-passo uni ou bicameral, atendidos no Laboratório de Marca-passo do Hospital das Clínicas da Universidade Federal de Minas Gerais (UFMG) no período de janeiro de 2001 a novembro de 2002, conforme descrito previamente $^{19}$. 0 projeto de pesquisa foi aprovado pelo Comitê de Ética em Pesquisa daUFMG e os pacientes foram esclarecidos a respeito do protocolo de estudo, tendo todos eles concordado na sua participação e assinado o termo de consentimento.

Foram incluídos pacientes consecutivos portadores de marca-passo modelo Affinity ${ }^{\circledR}$, fabricado pela St Jude Medical, com estimulação uni ou bicameral, com no mínimo três meses de implante do gerador e dos eletrodos, desde que aptos a realizarem o ecocardiograma, a monitorização por Holter de $24 \mathrm{~h}$, a radiografia de tórax, a sorologia para doença de Chagas e a telemetria do gerador. Foram excluídos apenas os pacientes impossibilitados de realizar os exames necessários. De cada paciente foi obtida ficha clínica incluindo os seguintes dados: nome, idade, sexo, classe funcional, indicação para implante de marca-passo, modo de estimulação e patologia de base. Os pacientes foram considerados chagásicos quando apresentavam pelo menos duas reações sorológicas positivas para a doença, por métodos distintos.

A monitorização eletrocardiográfica prolongada pelo sistema Holter foi realizada com o paciente mantendo suas atividades habituais. A gravação foi feita em gravadores modelo Cardioflash, marca Cardios e analisada em sistema computadorizado Burdick /DMI/ Cardios (São Paulo, Brasil), dotado de algoritmo que permite identificação das espículas de marca-passo. Os exames foram cuidadosamente revistos por um único pesquisador qualificado (VCVB) tendo sido quantificados, no período de 24 horas, o número total de extra-sístoles ventriculares e supraventriculares, o número de extra-sístoles ventriculares e supraventriculares isoladas, o número de extra-sístoles ventriculares e supraventriculares pareadas, os episódios de taquicardia ventricular não-sustentada (TVNS) e taquicardia ventricular sustentada (TVS), as taquicardias supraventriculares, os episódios de perda da captura atrial e/ou ventricular, e o número de pausas maior que dois segundos.

0 exame ecocardiográfico, disponível em 61 dos pacientes selecionados, foi realizado por técnica transtorácica em equipamento da marca Philips ATL HDI 5.000 (Bothell,
Washington, USA). 0 exame consistiu de avaliação pelos modos bidimensional, M e Doppler pulsátil, contínuo e em cores, seguindo-se as recomendações da Sociedade Americana de Ecocardiografia ${ }^{2}$. Foram avaliados o diâmetro diastólico final e a fração de ejeção do ventrículo esquerdo, obtida pelo método de Simpson. Este método não exige o pressuposto de que o ventrículo assemelhe-se geometricamente a um elipsóide, sendo fidedigno mesmo quando há distorções significativas da cavidade ventricular ${ }^{20}$. Todos os exames foram efetuados por um mesmo ecocardiografista experiente e que desconhecia a situação clínica e sorológica do paciente (MVLB).

A radiografia de tórax, disponível em 34 dos pacientes selecionados, foi feita no Serviço de Radiologia do Hospital das Clínicas da UFMG, com incidências póstero-anterior (PA) e perfil. A análise foi realizada de forma padronizada, com ênfase na medição do índice cardiotorácico e no local de fixação do eletrodo ventricular.

A análise do funcionamento do sistema artificial de estimulação cardíaca foi realizada em todos os pacientes através da telemetria, obtida com o programador APS II da Pacesseter, compatível com o marca-passo utilizado. A telemetria foi feita com o paciente em decúbito dorsal, colocando-se o cabeçote do programador sobre o gerador, possibilitando a comunicação entre o programador e o marca-passo. Em seguida, foi realizada a interrogação automática com obtenção de dados como o modo de estimulação, a freqüência cardíaca máxima e mínima programadas, a freqüência magnética, a impedância do gerador e do eletrodo, as funções de memória e o eletrograma intracavitário em tempo real. Após a interrogação do sistema de estimulação cardíaca, realizou-se a medida dos limiares de estimulação atrial e ventricular. Mensurou-se ainda a amplitude das ondas P e R em miliVolts, etapa necessária para a programação adequada das sensibilidades atrial e ventricular.

Análise estatística. Os dados foram descritos por proporções, no caso de variáveis qualitativas, e por média e desvio padrão (variáveis com distribuição normal) ou mediana e intervalo interquatil (variáveis sem distribuição normal). As comparações entre os grupos foram feitas pelo teste exato de Fisher, $t$ de Student e de Wilcoxon, de acordo com o tipo da variável e sua distribuição. O nível de significância estatística foi de 5\%. Foi calculado o coeficiente de correlação de Pearson entre a fração de ejeção do ventrículo esquerdo e o logaritmo neperiano do número de extra-sístoles ventriculares ao Holter. Com vistas ao estudo da relação entre a disfunção ventricular esquerda e a presença de ectopia ventricular ao Holter de 24 horas, a análise de covariância foi usada.

\section{RESULTADOS}

A amostra consistiu de 80 pacientes com marca-passo cardíaco, dos quais 45 (56\%) apresentavam sorologia positiva para T. cruzi. As características gerais da população 
estudada estão apresentadas na Tabela 1. Os pacientes chagásicos foram significativamente mais jovens $(55,9 \pm 12,8$ versus $68,3 \pm 15,0$ anos entre os não chagásicos, $\mathrm{p}<0,001$ ) e a distribuição por sexos foi diferente entre os grupos: predomínio de mulheres entre os chagásicos e de homens entre os não-chagásicos. A classe funcional (NYHA) não diferiu entre os dois grupos, sendo que quase a totalidade dos pacientes se apresentava em classes I ou II. Patologias como hipertensão arterial sistêmica, diabetes mellitus e doença coronariana foram mais freqüentes entre os nãochagásicos. 0 BAVT adquirido foi a indicação mais freqüente para o implante de marca-passo nos chagásicos (94\%) e nos não-chagásicos (76\%). A fração de ejeção do ventrículo esquerdo (FEVE) ao ecocardiograma foi significativamente menor nos pacientes chagásicos $(p=0,044)$, embora 0 diâmetro diastólico do VE e o índice cardiotorácico não apresentassem diferenças entre os grupos.

Os achados ao Holter de 24 horas estão sumariados na Tabela 2. As frequiências cardíaca média, máxima e mínima no período de gravação foram semelhantes nos grupos chagásico e não-chagásico. 0 número de batimentos cardíacos estimulados pelo marca-passo não diferiu nos dois grupos. Ritmo cardíaco predominantemente estimulado pelo marca-passo ocorreu em $87 \%$ dos pacientes não-chagásicos e em 94\% dos chagásicos (diferença estatisticamente não significativa). Disfunção de marca-passo ocorreu muito raramente no registro de 24 horas, não diferindo entre os dois grupos. Foram observadas falhas de captura atrial (um caso) e ventricular (um caso) e falhas de sensibilidade atrial (quatro casos) e ventricular (três casos).

A arritmia ventricular ao Holter de 24 horas foi mais frequiente entre os pacientes chagásicos, sendo estatisticamente significativa quanto ao número de extra-sístoles ventriculares $(\mathrm{p}=0,013)$. A extra-sistolia ventricular complexa, polimórfica, em pares ou episódios de taquicardia ventricular foi rara,

Tabela 1 - Características gerais dos 80 pacientes chagásicos e não-chagásicos portadores de marca-passo.

\begin{tabular}{|c|c|c|c|}
\hline Variável & $\begin{array}{l}\text { Não-chagásicos } \\
\qquad(\mathrm{n}=35)\end{array}$ & $\begin{array}{c}\text { Chagásicos } \\
(\mathrm{n}=45)\end{array}$ & Valor $\mathbf{p}$ \\
\hline Idade (anos) & $68(15)$ & $55(12)$ & $<0,001$ \\
\hline Sexo masculino (\%) & 56,0 & 30,0 & 0,023 \\
\hline HAS (\%) & 66,0 & 48,0 & 0,161 \\
\hline Diabetes mellitus (\%) & 16,0 & 5,0 & 0,233 \\
\hline Doença coronariana (\%) & 25,0 & 3,0 & 0,017 \\
\hline Pós-operatório (\%) & 18,0 & 3,0 & 0,078 \\
\hline Classe funcional I/II NYHA (\%) & 96,0 & 91,0 & 0,622 \\
\hline BAVT (\%) & 94,0 & 76,0 & 0,054 \\
\hline Rx tórax (n) & $27 / 35$ & $34 / 45$ & \\
\hline ICT ao Raio X de tórax (n=34) & $0,53(0,05)$ & $0,56(0,07)$ & 0,221 \\
\hline Ecocardiograma (n) & $27 / 35$ & $34 / 45$ & \\
\hline Fração ejeção VE & $0,60(0,15)$ & $0,55(0,20)$ & 0,044 \\
\hline Diâmetro diastólico final VE & $49,7(13,4)$ & $54,0(9,1)$ & 0,244 \\
\hline
\end{tabular}

Tabela 2 - Características ao Holter de 24 h dos pacientes chagásicos e nãochagásicos portadores de marca-passo.

\begin{tabular}{lccc}
\hline Variável & $\begin{array}{c}\text { Não-chagásico } \\
(\mathbf{n}=\mathbf{3 5})\end{array}$ & $\begin{array}{c}\text { Chagásico } \\
(\mathbf{n}=\mathbf{4 5})\end{array}$ & Valor $\mathbf{p}^{* *}$ \\
\hline FC média (bpm) & $72(8)$ & $69(8)$ & 0,100 \\
FC mínima (bpm) & $54(6)$ & $54(6)$ & 0,729 \\
FC máxima (bpm) & $118(27)$ & $108(14)$ & 0,197 \\
ES ventriculares (n) & $29(441)$ & $435(1580)$ & 0,013 \\
ES supraventriculares (n) & $0(0)$ & $0(1)$ & 0,090 \\
Batimentos estimulados pelo marca-passo (\%) & 87 & 94 & 0,124 \\
\hline
\end{tabular}

Dados expressos em proporções ou pela mediana (distância interquartílica). $\mathrm{FC}=$ frequiência cardíaca; $\mathrm{ES}=$ extra-sístoles.

embora não significativamente mais freqüente entre os pacientes chagásicos. As extra-sístoles supraventriculares também ocorreram raramente e sem diferença estatisticamente significativa entre os dois grupos.

Na Figura 1, observa-se o diagrama de dispersão entre a fração de ejeção do ventrículo esquerdo e o logaritmo neperiano do número de extra-sístoles ventriculares ao Holter de 24 horas: observou-se uma forte correlação entre as variáveis, com coeficiente de correlação de Pearson de $-0,45$ $(\mathrm{p}<0,001)$. Quando se utiliza a análise de covariância, observa-se que a densidade dessas extra-sistoles se relaciona mais fortemente com a FEVE $(\mathrm{p}=0,006)$ do que à presença de sorologia positiva para doença de Chagas $(p=0,059)$.

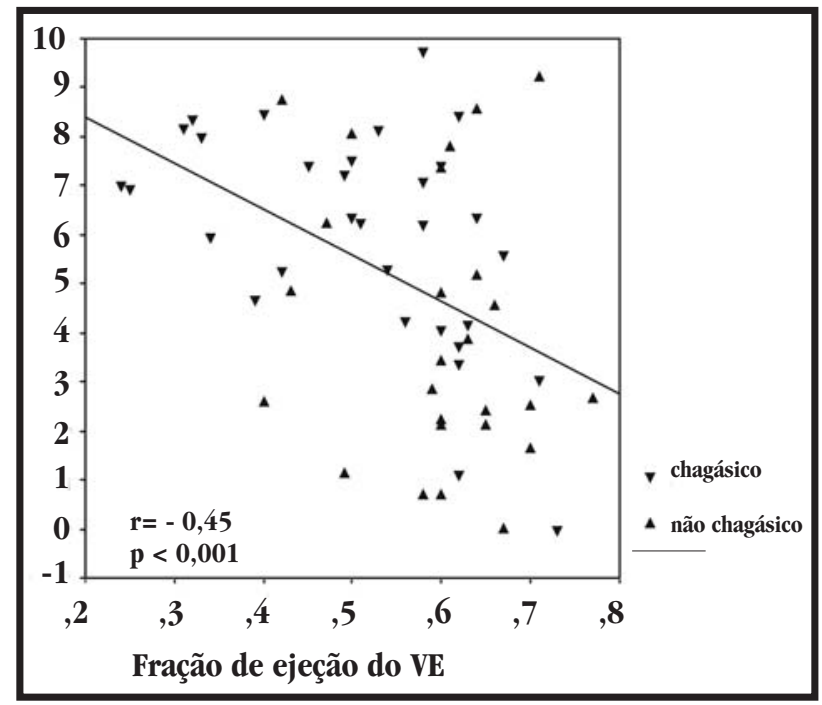

Figura 1 - Diagrama de dispersão entre a fração de ejeção do ventrículo esquerdo e o logaritmo neperiano do número de extra-sístoles ventriculares ao Holter $24 \mathrm{~h}$.

Os dados referentes ao marca-passo estão descritos na Tabela 3. 0 modo de estimulação, o tempo desde o implante e os limiares de estimulação atrial e ventricular não diferiram entre os dois grupos. Em $62 \%$ dos pacientes chagásicos e $78 \%$ dos não-chagásicos o eletrodo ventricular estava posicionado em região subtricuspídea do ventrículo direito. Todos os eletrodos do grupo chagásico e 89\% dos nãochagásicos tinham corticóide na ponta. 
Tabela 3 - Características da estimulação cardíaca artificial dos pacientes chagásicos e não-chagásicos portadores de marca-passo.

\begin{tabular}{lccc}
\hline Variável & Não-chagásicos & Chagásicos & Valor p \\
\hline Modo DDD (\%) & 48,0 & 44,0 & 0,761 \\
Tempo de implante (meses) & $14(3)$ & $14(3)$ & 0,764 \\
Limiar V & $0,75(0,25)$ & $0,75(0,25)$ & 0,284 \\
Limiar A & $0,75(0,25)$ & $0,75(0,50)$ & 0,907 \\
Onda R evocada & $11,2(4,7)$ & $12,2(7,0)$ & 0,810 \\
Eletrodo com esteróide & 100 & 89 & 0,127
\end{tabular}

Dados expressos pela média (desvio padrão) ou pela proporção. DDD marca-passo de dupla câmara, Limiar V = limiar de estimulação ventricular, Limiar A = limiar de estimulação atrial.

\section{DISCUSSÃ0}

Na América Latina a doença de Chagas é uma importante causa de lesão do tecido de condução do estímulo elétrico cardíaco e o surgimento de vários tipos de arritmia. Estudos comparativos entre a miocardiopatia chagásica e outras miocardiopatias revelam que pacientes chagásicos apresentam maior comprometimento do ventrículo esquerdo e maior incidência de arritmias, incluindo arritmias graves, apresentando portanto pior prognóstico ${ }^{9}$.

Embora existam na literatura estudos que analisaram as características clínicas de pacientes chagásicos com marca-passo cardíaco, nenhum estudo comparou pacientes chagásicos e nãochagásicos portadores de marca-passo considerando-se a fração de ejeção ao ecocardiograma, a incidência de arritmias ventriculares ao Holter e o limiar de estimulação ventricular à telemetria. Com o intuito de avaliar estas características dos pacientes chagásicos e não-chagásicos portadores de marcapasso foram selecionados 80 dos aproximadamente 2000 pacientes portadores de marca-passo cardíaco que são regularmente acompanhados no Laboratório de Marca-Passo do Hospital das Clínicas da UFMG.

Em nosso estudo, a idade média do paciente chagásico $(55,9 \pm 12,8$ anos $)$ foi significativamente menor quando comparada ao não-chagásico (68,32 $\pm 14,9$ anos). Achado semelhante ao estudo de Costa e cols no qual foram comparadas características clínicas e epidemiológicas de pacientes chagásicos e não-chagásicos submetidos a implante de marca-passo cardíaco. Considerou-se, neste estudo, que o fato do grupo chagásico ser mais jovem tinha implicações importantes, sendo o responsável pelo maior número de reoperações nestes pacientes ${ }^{8}$.

A população aqui estudada caracterizou-se ainda por apresentar poucos sintomas cardiovasculares, como demonstrado pela classificação funcional da New York Heart Association (NYHA). A maioria dos pacientes chagásicos e nãochagásicos encontrava-se nas classes funcionais I e II (NYHA) não havendo diferença entre os dois grupos. Bestetti e cols comparando a evolução clínica de pacientes com cardiopatia chagásica e miocardiopatia dilatada não-chagásica, observaram que os dois grupos apresentavam insuficiência cardíaca leve a moderada, não diferindo quanto à classificação funcional da $\mathrm{NYHA}^{3}$. Assim como neste estudo, nossos pacientes estavam em uso de IECA; além desse tipo de medicamento, esses últimos freqüentemente usavam digital e diurético, o que pode ter contribuído para diminuição dos sintomas cardiovasculares.

0 distúrbio do ritmo mais freqüente entre os pacientes que se submeteram ao implante de marca-passo foi o BAVT (53 dos 80 pacientes), sendo que a doença do nó sinusal ocorreu em outros 10 pacientes. Nos 17 pacientes restantes, não foi possível obter esta informação. Apesar de não ter ocorrido diferença estatisticamente significativa entre os dois grupos, o distúrbio de condução mais freqüentemente relacionado ao implante de marca-passo entre os chagásicos foi o BAVT (34 em 43 pacientes), confirmando os achados de Leão e cols, que analisaram o perfil de pacientes chagásicos e não-chagásicos do Registro Brasileiro de Marca-passos ${ }^{12}$.

A disfunção ventricular esquerda é reconhecida como importante preditor de mortalidade na cardiopatia chagásica crônica ${ }^{4}$, sendo a ecocardiografia um método de excelente confiabilidade na avaliação sisto-diastólica do ventrículo esquerdo. No presente estudo, a avaliação ecocardiográfica com medida da fração de ejeção e diâmetro diastólico final do ventrículo esquerdo foi realizada em 61 pacientes: 34 chagásicos e 27 não-chagásicos. Verificou-se que a média do diâmetro diastólico final, calculada para os dois grupos, não apresentou diferença estatisticamente significativa. No entanto, a fração de ejeção foi significativamente menor no grupo chagásico, observação esta semelhante a de outros autores que encontraram importante comprometimento da função ventricular esquerda na cardiopatia chagásica, considerando esta alteração um importante preditor de sobrevida ${ }^{1}{ }^{14}$

As arritmias ventriculares constituem um dos fenômenos mais expressivos da cardiopatia chagásica crônica por estarem relacionadas à morte súbita, sendo que as extra-sístoles ventriculares juntamente com os distúrbios da condução intraventricular constituem as primeiras manifestações eletrocardiográficas do envolvimento ventricular na doença de Chagas $^{15}$. Existem evidências de que nos pacientes com acometimento miocárdico, o grau de disfunção do ventrículo esquerdo é um dos fatores determinantes para a ocorrência e a complexidade da arritmia ventricular: quanto maior a disfunção ventricular, mais freqüentes e mais complexas são as arritmias encontradas ao Holter $24 \mathrm{~h}$, correlação esta mais expressiva nos pacientes chagásicos ${ }^{5}$.

No presente estudo, o número médio de extra-sístoles ventriculares isoladas em $24 \mathrm{~h}$ foi significativamente maior no grupo chagásico; verificou-se ainda relação significativa entre a disfunção sistólica do ventrículo esquerdo, medida através da fração de ejeção, e a presença de arritmia ventricular (extra-sistolia) ao Holter $24 \mathrm{~h}$.

Dentre os parâmetros disponíveis para avaliação do funcionamento do sistema de estimulação cardíaca artificial, a determinação dos limiares de estimulação atrial e ventricular é um dos mais importantes, tanto em cirurgias de implante como no acompanhamento do paciente com marca-passo cardíaco. Alguns autores, levando em consideração o fato de que a ponta do ventrículo direito (local onde normalmente é fixado o eletrodo 
do marca-passo) no paciente chagásico apresenta lesões como espessamento endocárdico e trombose, compartilham a idéia de que os limiares de estimulação ventricular são mais elevados nos chagásicos quando comparados aos não-chagásicos ${ }^{17}$.

Diante do exposto, Korman e Jatene sugeriram uma nova posição para 0 eletrodo endocavitário em ventrículo direito, a posição subtricuspídea. Gauch e cols verificaram, em um estudo comparativo do limiar de estimulação agudo e crônico entre pacientes chagásicos e não-chagásicos com eletrodo fixado em posição subtricuspídea, não haver diferença significativa dos limiares de estimulaçã̃ $0^{10}$. Em nossa amostra, $61,9 \%$ dos pacientes chagásicos e 77,8\% dos não-chagásicos tinham os eletrodos ventriculares fixados em posição subtricuspídea. Ainda, $100 \%$ dos pacientes chagásicos e 89,5\% dos não-chagásicos eram portadores de eletrodo endocavitário com esteróide na ponta. Semelhantemente ao que foi verificado por Gauch e colaboradores não encontramos diferença significativa no limiar de estimulação ventricular entre os pacientes chagásicos e nãochagásicos.

Considerando-se os achados de menor fração de ejeção do ventrículo esquerdo e maior incidência de arritmia ventricular em 24 horas nos pacientes chagásicos com marca-passo, quando comparados a pacientes não-chagásicos, e tendo em vista dados da literatura que relacionam estes achados com pior prognóstico, é possível que os pacientes chagásicos com marca-passo apresentem maior risco de morte do que pacientes nãochagásicos com o mesmo dispositivo.

\section{REFERÊNCIAS BIBLIOGRÁFICAS}

1. Acquatella H, Schiller NB, Puigbo JJ, Giordano H, Suarez JA, Casal H, Arreaza N, Valecillos R, Hirschhaut E. M-mode and two dimensional echocardiography in chronic Chagas'disease. A clinical and pathologic study. Circulation 62:787-799, 1980.

2. American Society of Echocardiography Committee on Standars, Subcomittee on Quantitation of Two-Dimensional Echocardiograms: Recommendations for quantitation of the left ventricle by two-dimensional echocardiography. Journal of American Society of Echocardiografy 2:361-367, 1989.

3. Bestetti RB, Muccilo G. Clinical course of Chagas' disease: a comparison with dilated cardiomyopathy. International Journal of Cardiology 60: 187193,1997.

4. Bestetti RB, Rossi MA. A rationale approach of mortality risk stratification in Chagas' heart disease. International Journal of Cardiology 58: 199-209, 1997.

5. Carrasco HA, Uerro L, Parada H, Molina C, Vegas E, Chuecos R. Ventricular arrhytmias and left ventricular myocardial function in chronic chagasic patients. International Journal of Cardiology 28:35-41, 1990.
6. Carrasco HA, Parada H, Guerrero L, Duque M, Duran D, Molina C. Prognostic implication of clinical, electrocardiographic and hemodynamic findings in chronic Chagas'disease. International Journal of Cardiology 43: 27-38, 1994.

7. Chagas C, Villela E. Forma cardíaca da trypanosomíase americana. Memórias do Instituto Oswaldo Cruz 14: 5-61,1992.

8. Costa R, Rassi A, Leão MIP Estudo clínico e epidemiológico de pacientes submetidos a implante de marcapasso cardíaco artificial permanente: comparação dos portadores de doença de Chagas com os de doenças degenerativas do sistema de condução. Revista Brasileira de Cirurgia Cardiovascular 19: 107-114, 2004.

9. Freitas H, Chizzola PR, Markus MRP, Cuoco MAR, Paes AT, Lima ACP, Bocchi EA, Mansur AJ. Prognosis in severe heart failure due to Chagas'disease worse than heart failure due to idiophatic, ischaemic or hypertensive cardiomyopathy. European Heart Journal 4: 451, 2002.

10. Gauch PR, Kormann DS, Kormann SJ, Jatene AD. Estudo comparativo do limiar de estimulação agudo e crônico em portadores de doença de Chagas e miocardiosclerose com marca-passo cardíaco. Arquivos Brasileiros de Cardiologia 35: 359-361, 1980

11. Kormann DS, Jatene AD. Triângulo eletro-vértebro-diafragmático no posicionamento de eletrodo endocavitário para marcapassos cardíacos. Arquivos Brasileiros de Cardiologia 30 (supl 2): 380, 1997.

12. Leão MIP, Costa R, Pachón-Mateos JC, Galvão Filho SS, Takeda RT. Registro brasileiro de marca-passos no ano de 1995: análise do perfil dos pacientes chagásicos e não-chagásicos. Reblampa 9: 75-82, 1996.

13. Lopes ER, Chapadeiro E. Patogenia das manifestações cardíacas na doença de Chagas. Arquivos Brasileiros de Cardiologia 65: 367- 375, 1995.

14. Mady C, Cardoso R, BarretoA, DA Luz PL, Bellotti G, Pillegi F. Survival and predictors of survival and predictors of survival in patients with congestive heart failure due to Chagas'cardiomyopathy. Circulation 90: 3098-3102, 1994.

15. Maguire JH, Ramos NB, Santana 00. Comparação do eletrocardiograma convencional com o eletrocardiograma dinâmico na doença de Chagas. Arquivos Brasileiros de Cardiologia 37:82, 1981.

16. Martinelli Filho M, Siqueira SF, Moreira H, Fagundes A, Pedrosa A, Nishioka SD, Costa R, Scanavacca M, D'Avila A, Sosa E. Probability of occurence of life-threatening ventricular arrhytmias in Chagas'disease versus non-Chagas disease. Pace 23: 1944-1946, 2000.

17. Naime A, Bello A, Jaen R. Permanent cardiac pacing experience with arrhythmias caused by Chagas' disease and other cardiopathies. Journal of Cardiovascular Surgery 13:560-567, 1972.

18. Parada H, Carrasco HG, Guerrero L, Molina C, Checos R, Martinez 0. Diferencias clínicas y paraclínicas entre la miocardiopatia chagásica cronica y las miocardiopatias dilatadas primarias. Arquivos Brasileiros de Cardiologia 3: 99-104,1989.

19. Ribeiro AL, Rincon LG, Oliveira BG, Vinha CR, Melatto D, Torres AA, Barros MVC, Levine PA. Automatic adjustment of pacing output in the clinical setting. American Heart Journal 147: 127-131, 2004.

20. Vuille C, Weyman AE. Left Ventricle I: General Considerations, Assessment of Chamber Size and Function. In: Weyman AE (ed) Principles and Pratice of Echocardiography. Lea \& Febiger, USA, p.575-611, 1994.

21. World Health Organization. Infectious diseases. Chagas disease. Disponível em http://www.who.int/ctd/chagas/whodocs.htm, 1997. 